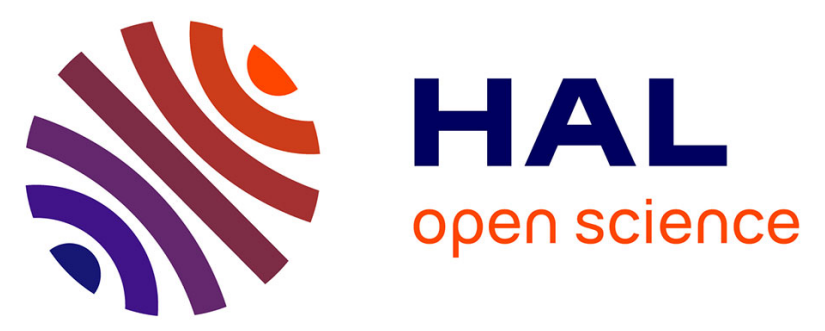

\title{
Multiconfigurational time-dependent Hartree calculations for tunneling splittings of vibrational states: Theoretical considerations and application to malonaldehyde
}

Thorsten Hammer, Mauricio D. Coutinho-Neto, Alexandra Viel, Uwe Manthe

\section{- To cite this version:}

Thorsten Hammer, Mauricio D. Coutinho-Neto, Alexandra Viel, Uwe Manthe. Multiconfigurational time-dependent Hartree calculations for tunneling splittings of vibrational states: Theoretical considerations and application to malonaldehyde. Journal of Chemical Physics, 2009, 131 (22), pp.4109-1-13. 10.1063/1.3272610 . hal-00667038

\section{HAL Id: hal-00667038 \\ https://hal.science/hal-00667038}

Submitted on 10 Jul 2017

HAL is a multi-disciplinary open access archive for the deposit and dissemination of scientific research documents, whether they are published or not. The documents may come from teaching and research institutions in France or abroad, or from public or private research centers.
L'archive ouverte pluridisciplinaire HAL, est destinée au dépôt et à la diffusion de documents scientifiques de niveau recherche, publiés ou non, émanant des établissements d'enseignement et de recherche français ou étrangers, des laboratoires publics ou privés. 


\title{
Multiconfigurational time-dependent Hartree calculations for tunneling splittings of vibrational states: Theoretical considerations and application to malonaldehyde
}

\author{
Thorsten Hammer, ${ }^{1, a)}$ Mauricio D. Coutinho-Neto, ${ }^{2}$ Alexandra Viel, ${ }^{3}$ and Uwe Manthe ${ }^{1, b)}$ \\ ${ }^{1}$ Theoretische Chemie, Fakultät für Chemie, Universität Bielefeld, Universitätsstr. 25, \\ D-33615 Bielefeld, Germany \\ ${ }^{2}$ Centro de Ciências Naturais e Humanas, Universidade Federal do ABC, Rua Santa Adélia, \\ 166 Santo André, São Paulo BR-09.210-170, Brazil \\ ${ }^{3}$ Institut de Physique de Rennes (IPR), CNRS UMR 6251, Université de Rennes 1, Campus de Beaulieu, \\ F-35042 Rennes, France
}

(Received 21 September 2009; accepted 18 November 2009; published online 9 December 2009)

\begin{abstract}
Full-dimensional multiconfigurational time-dependent Hartree calculations on the tunneling splitting of the vibrational ground state and the low lying excited states of malonaldehyde are presented. Methodological developments utilizing the symmetry of double well systems for the efficient calculation of tunneling splittings are described and discussed. Important aspects of the theory underlying the previously communicated results for the ground state tunneling splitting [M. D. Coutinho-Neto et al., J. Chem. Phys. 121, 9207 (2004)] are detailed and further developments facilitating the calculation of tunneling splittings for vibrationally excited states are introduced. Utilizing these developments, the 14 lowest vibrational states of malonaldehyde, i.e., seven tunneling splittings, have been computed. The tunneling splittings are found to vary significantly depending on the particular vibrational excitation. This results in a complex pattern of vibrational levels. Studying the dependence of the tunneling splittings on the vibrational excitation, good agreement with available experimental results is found and intuitive interpretations of the results can be given. (C) 2009 American Institute of Physics. [doi:10.1063/1.3272610]
\end{abstract}

\section{INTRODUCTION}

Proton transfer processes are ubiquitous in chemistry and play an important role in many processes of biochemical and biological interest. Due to the light mass of the proton, quantum mechanical tunneling plays often a central part in these processes. Focusing particularly on intramolecular proton transfer and the quest for its accurate theoretical description, malonaldehyde has been a benchmark system for about 3 decades. Exhibiting two equivalent tautomeric forms, the potential energy surface (PES) of the system presents a symmetric double well structure with a relatively small barrier of approximately $4 \mathrm{kcal} / \mathrm{mol} .{ }^{1}$ This small barrier gives rise to large splittings of the vibrational states.

Experimentally, the tunneling splitting of the ground vibrational state has been accurately measured already by a series of experiments in the 1980s (Refs. 2-5) and further work $^{6,7}$ finally yielded the exceedingly accurate value of $21.5831383(6) \mathrm{cm}^{-1}$. In contrast, the measurement of tunneling splittings of the vibrationally excited states is an open and still developing field. Only few experiments on these excited state splittings have been published. ${ }^{8-11}$ The number of vibrationally excited states investigated is rather limited and the accuracy of the measured data is many orders of magnitude lower than that for the ground state tunneling splitting.

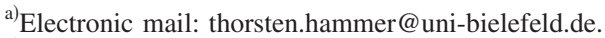

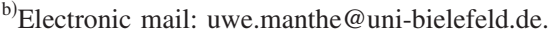

Theoretically, the accurate description of the proton tunneling in malonaldehyde has been found to be a challenging question requiring a truly multidimensional description of the underlying quantum dynamics. Early theoretical work resorted to reaction surface descriptions ${ }^{12,13}$ or semiclassical calculations. ${ }^{14-18}$ In these studies, varying agreement between the computed tunneling splittings and the experimental values was found. However, due to the restrictions in the quality of the $a b$ initio data available at that point in time, agreement with experiment could hardly have been expected to be more than fortuitous. Furthermore demonstrating the complexity of the dynamical problem, imaginary time path integral calculations showed that the quantum mechanical tunneling is not restricted to the motion of the hydrogen atom alone but is coupled to the motion of the heavy backbone atoms. ${ }^{19}$ Following the earlier work and utilizing high level $a b$ initio calculations which then became available, Tautermann et al. ${ }^{20,21}$ and Mil'nikov et al. ${ }^{22,23}$ combined the semiclassical instanton approach with an on-the-fly computation of the electronic energies at a reasonably high level of $a b$ initio theory and computed ground state tunneling splittings in good or even perfect agreement with experiment. However, at that point it remained an open question whether this agreement was just a result of a fortuitous cancellation of errors resulting from the semiclassical approximations and the inaccuracies of the $a b$ initio data.

Significant progress was achieved when Yagi et al. ${ }^{24}$ constructed the first full 21-dimensional ab initio PES for malonaldehyde using modified Shepard interpolation 
techniques $^{25,26}$ and $a b$ initio data at the MP2/6-31G(d,p) level. While the limitations in the accuracy of the $a b$ initio data, e.g., the barrier height on this level, $3.6 \mathrm{kcal} / \mathrm{mol}$, is about $0.4 \mathrm{kcal} / \mathrm{mol}$ too low, still prevented perfect agreement with experiment, the PES is sufficiently accurate to allow a detailed investigation of the effects of different dynamical approximations. Instanton calculations for this PES by Mil'nikov et al. $^{23}$ yielded a ground state tunneling splitting of $30.7 \mathrm{~cm}^{-1}$. Based on this PES, then the first fulldimensional quantum calculations of the ground state tunneling splitting in malonaldehyde could be published. ${ }^{27,28} \mathrm{~A}$ first short communication ${ }^{27}$ reported results independently obtained using two different, numerically exact methods: a diffusion Monte Carlo (DMC) approach using projection operator imaginary time spectral evolution ${ }^{29}$ (POITSE) and an imaginary time wave packet propagation approach utilizing the multiconfigurational time-dependent Hartree $^{30,31}$ (MCTDH) method. Ground state splittings of $25.7 \pm 0.3 \mathrm{~cm}^{-1}$ and $25 \mathrm{~cm}^{-1}( \pm 10 \%)$, respectively, were found. While the details of POITSE calculations have been described in a subsequent paper, ${ }^{28}$ which also reported results on the isotopically substituted malonaldehyde, a detailed description of the techniques used in the MCTDH calculations of the tunneling splitting has not yet been given. Presenting and discussing these details is one of the aims of the present article.

Before continuing our introduction toward the specific aims of the present work, very recent further progress toward an accurate description of the malonaldehyde system should be mentioned. Utilizing recent progress in electronic structure theory and the construction of PESs, Wang et al. ${ }^{1}$ presented a significantly more accurate PES. Their PES construction employed the permutationally invariant polynomial approach developed by Braams and Bowman. ${ }^{32}$ Using a fixed node DMC approach, Wang et al. calculated a tunneling splitting of $21-22 \mathrm{~cm}^{-1}$ for their new PES. Given the present limits even of elaborous state of art electronic structure calculations, the agreement between this theoretical result and experiment is excellent. However, since this new PES was not yet available to us while most of the extensive quantum dynamics calculations presented here have been performed, the work presented in the following still relies on the older PES of Yagi et al. In this context, also calculations by Tew et al. ${ }^{33}$ using a $a b$ initio based reaction surface PES and a vibrational configuration interaction (VCI) approach should be mentioned.

The aim of the present work is twofold. First ideas facilitating a particularly efficient description of double well systems within the MCTDH approach are presented and discussed. In this context, the methods used in previous work ${ }^{27}$ are described in full detail. Moreover, further improvements in the theoretical concept are presented. Recent developments in MCTDH techniques, ${ }^{34}$ which can be utilized to further improve the efficiency of the calculations, are incorporated into the approach. While MCTDH calculations presented in the previous communication ${ }^{27}$ have been restricted to the computation of the ground state tunneling splitting, these developments now increase the efficiency of the MCTDH calculation sufficiently to also facilitate calculations of the tunneling splittings of low lying vibrationally excited states.

The investigation of the tunneling splittings of vibrationally excited states is the second major aim of the present work. Rigorous full-dimensional quantum dynamics calculations on these quantities are published for the first time. Since the POITSE DMC calculations, which provided data of superior accuracy for the ground state tunneling splitting, cannot be used to compute excited state tunneling splittings, the development of the efficient MCTDH techniques has been particularly important in this context. Based on these results, the dependence of the tunneling splitting on the particular vibrational mode excited is discussed. While the inaccuracies of the particular PES employed might still limit the quantitative accuracy of the computed data, the ground state tunneling splitting on this PES deviates by about $4 \mathrm{~cm}^{-1}$ or $20 \%$ from the accurate (experimental) value, the conclusions drawn can be expected to be valid on an at least semiquantitative level.

The article is organized as follows: In Sec. II the MCTDH method is reviewed. The two different approaches for the calculation of eigenstates used in the previous communication $^{27}$ and in the present work are described. Section III discusses the difficulties arising when treating symmetric double well systems within the MCTDH approach and presents two different approaches for their solution. Section IV details the system description and the specifics of the MCTDH calculations. The results are presented in Sec. V and discussed in Sec. VI. The article closes with a conclusions and perspectives section.

\section{CALCULATION OF VIBRATIONAL STATES USING MCTDH}

\section{A. MCTDH}

The MCTDH method (Refs. 30 and 31) has proven to be an efficient approach for the quantum dynamical description of high-dimensional systems. ${ }^{35-38}$ The method is based on the following ansatz:

$$
\psi\left(x_{1}, \ldots, x_{f}, t\right)=\sum_{j_{1}=1}^{n_{1}} \cdots \sum_{j_{f}=1}^{n_{f}} A_{j_{1}, \ldots, j_{f}}(t) \prod_{\kappa=1}^{f} \phi_{j_{\kappa}}^{(\kappa)}\left(x_{\kappa}, t\right) .
$$

The correlated multidimensional wave function is represented employing time-dependent expansion coefficients $A_{j_{1}, \ldots, j_{f}}$ and a basis of time-dependent single-particle functions (SPFs) $\phi_{i}^{(\kappa)}$. The time-dependent (one-dimensional) SPFs are represented on an underlying time-independent basis or grid using, e.g., a discrete variable representation ${ }^{39-41}$ (DVR) or fast Fourier transform ${ }^{42}$ (FFT) techniques. Equations of motion are derived employing the Dirac-Frenkel variational principle. The correlation DVR (CDVR) scheme $^{43}$ can be employed to calculate potential energy matrix elements appearing in the equations of motion for arbitrary potentials.

The calculation of vibrational states within the MCTDH approach is based on propagation in imaginary time. In the previously communicated work on the malonaldehyde 
system ${ }^{27}$ and in the present work, two different schemes have been employed. An approach based on an iterative Lanczostype diagonalization ${ }^{44}$ has been used in the older calculations while a more recently developed scheme based on simultaneous relaxation of a set of wave functions ${ }^{34}$ is used for the present calculations. Since the present article intends to describe the underlying theory of MCTDH calculations for double well systems used in the previous communication as well as in the new present calculations, both approaches will be briefly described in Secs. II B and II C to provide a proper basis for the further discussion.

\section{B. Iterative Lanczos-type diagonalization}

In the iterative diagonalization approach, ${ }^{44}$ an orthonormal basis $\left\{\left|\psi_{0}\right\rangle, \ldots,\left|\psi_{M}\right\rangle\right\}$ is constructed by the recursion

$$
\left|\psi_{m}\right\rangle=C\left(\mathrm{e}^{-\beta \hat{H}}\left|\psi_{m-1}\right\rangle-\sum_{i=0}^{m-1} \hat{P}\left|\psi_{i}\right\rangle \alpha_{i}\right) .
$$

Here $C$ is a normalization constant and $\hat{P}$ is a projector on the space spanned by the SPFs of the wave function $\mathrm{e}^{-\beta \hat{H}}\left|\psi_{m-1}\right\rangle$. Within the above recursion, the action of $\mathrm{e}^{-\beta \hat{H}}$ is evaluated using the MCTDH propagation in imaginary time for a time interval given by the parameter $\beta$. The $\alpha_{i}$ are obtained by solving a system of linear equations,

$$
\left\langle\hat{P} \psi_{j} \mid \mathrm{e}^{-\beta \hat{H}} \psi_{m-1}\right\rangle=\sum_{i=0}^{m-1}\left\langle\hat{P} \psi_{j} \mid \hat{P} \psi_{i}\right\rangle \alpha_{i}, \quad j=0, \ldots, m-1 .
$$

Starting with an initial wave function $\left|\psi_{0}\right\rangle$, Eq. (2) is used to generate a set of wave functions $\left\{\left|\psi_{0}\right\rangle, \ldots,\left|\psi_{M}\right\rangle\right\}$. Then, the $\mathrm{e}^{-\beta \hat{H}}$ operator is diagonalized in this basis to obtain eigenvalues and eigenfunctions of the Hamiltonian. The procedure can be repeated with increased values of $M$ until convergence for the required number of eigenstates is reached. A more detailed description of the approach discussing also the relation to other theoretical approaches is given in Ref. 44 .

This scheme has been successfully applied to the calculation of small to moderate numbers of vibrational states and has been used in previous work on malonaldehyde. ${ }^{27,44,45}$ Difficulties arise when large numbers of states need to be calculated since the corresponding large number of iterations typically requires increasingly large SPF bases. Since the computational effort scales approximately proportional to the number of configurations in the SPF basis, the number of accessible states is very limited. Due to these limitations, in our previous study ${ }^{27}$ only the ground state tunneling splitting could be calculated accurately.

\section{Block relaxation of state-averaged MCTDH wave functions}

While the iterative Lanczos-type diagonalization approach strongly restricts the number of accessible vibrational states, the recently introduced state-averaged $\mathrm{MCTDH}$ approach $^{34}$ enables the calculation of larger numbers of vi- brational states. Within this approach, a set of multiple wavepackets is described within a common SPF basis. The wavefunction ansatz reads

$$
\begin{aligned}
\psi_{m}\left(x_{1}, \ldots, x_{f}, t\right)= & \sum_{j_{1}=1}^{n_{1}} \ldots \sum_{j_{f}=1}^{n_{f}} A_{j_{1}, \ldots, j_{f}, m}(t) \prod_{\kappa=1}^{f} \phi_{j_{\kappa}}^{(\kappa)}\left(x_{\kappa}, t\right), \\
& m=1, \ldots, n_{\text {packet }} .
\end{aligned}
$$

Here $n_{\text {packet }}$ wave functions labeled $\psi_{m}$ are represented. As in Eq. (1), the $A_{j_{1}, \ldots, j_{f} m}$ denote time-dependent expansion coefficients and the $\phi_{j_{\kappa}}^{(k)}$ denote time-dependent SPFs. Comparing to Eq. (1), the expansion coefficients $A_{j_{1}, \ldots, j_{f}, m}$ show an additional index $m$ that specifies the corresponding wave function $\psi_{m}$. The SPFs do not carry such an additional index since the state-averaged MCTDH approach employs a single common basis of SPFs $\phi_{j_{\kappa}}^{(\kappa)}$.

Representing multiple wave functions within a common set of SPFs has several advantages. In comparison to multiple separate calculations involving only a single wavepacket, the numerical effort of a MCTDH calculation can be reduced. The savings in numerical effort can be quite significant in calculations employing the CDVR scheme to evaluate potential energy matrix elements. Another advantage is particularly important for the calculation of eigenstates: As different wave functions are represented in the same SPF basis in the state-averaged MCTDH approach, linear combinations of the wave functions can be computed efficiently and a scheme to calculate vibrational eigenstates based on block relaxation can be devised. ${ }^{34}$ This approach is based on repeated steps which consist of an application of the $\mathrm{e}^{-\beta \hat{H}}$ operator on the state-averaged MCTDH wave function followed by a subsequent orthonormalization of the resulting sets of wave functions.

The detailed scheme works as follows: Starting from an initially chosen set of wavepackets represented within the state-averaged MCTDH ansatz, an iteration sequence is started. In an iteration step, first the $\mathrm{e}^{-\hat{\beta H}}$ operator is applied, i.e., an imaginary time propagation is performed. Then the resulting set of wave functions is Gram-Schmidt orthonormalized. The matrix representation of the Hamiltonian operator in this basis is constructed and diagonalized. Concluding the iteration step, the resulting eigenvectors are then used to transform the set of states to their (approximate) eigenstate representation. These iteration steps are repeated until convergence is reached.

With this new scheme, larger numbers of vibrational states can be calculated. Thus, this new scheme is found superior to the previously employed Lanczos-type iterative diagonalization approach and is employed in the new calculations presented here. A more detailed description of the approach discussing its efficiency and its relation to other approaches is given in Ref. 34.

\section{SYMMETRIC PROTON TRANSFER}

\section{A. General ideas}

Although the MCTDH approach is designed for the treatment of high-dimensional systems, malonaldehyde with 


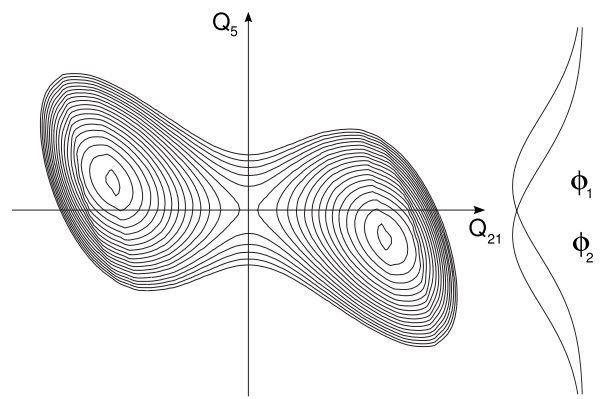

FIG. 1. Contour plot of the PES of malonaldehyde for a two-dimensional cut along the reaction coordinate $\left(Q_{21}\right)$ and an important antisymmetric coordinate $\left(Q_{5}\right)$. An intuitive (nonorthogonal) basis required to qualitatively describe the dynamics in the antisymmetric coordinate is indicated.

its 21 internal degrees of freedom still posts a significant challenge. However, the symmetry of the symmetric proton transfer system can be utilized to increase the efficiency of the MCTDH treatment. To illustrate the underlying idea, Fig. 1 shows a two-dimensional plot of a double well system considering the reaction coordinate and a coupled antisymmetric coordinate. The figure indicates a minimal basis in the coupled antisymmetric coordinate (SPFs could be obtained by orthogonalization of the depicted functions) required to represent the ground state wave function for this system. Since the ground state is localized equivalently in both wells, at least two different SPFs are required in either of the two coordinates to describe the wave function qualitatively correct. At this point one should remember that the numerical effort of a MCTDH calculation scales approximately proportional to the number of configurations: The numerical effort is approximately proportional to $n^{f}$ if $n$ SPFs are used in each of the $f$ degrees of freedom. Thus, for a symmetric double well system with $f-1$ coordinates coupled to the reaction coordinate in the way described above, the resulting number of configurations in the minimal basis would be $2^{f}$. Taking $f=21$ as the simplest possible estimate (malonaldehyde has 21 internal degrees of freedom), an estimate for the lower boundary of the number of configurations required for a qualitatively meaningful description amounts to $2^{21}$ $=2097152$. Thus, a quantitatively correct description of the malonaldehyde system clearly could not be achieved using such a straightforward approach.

At this point it is important to note that the above described problem can partially be avoided by utilizing the symmetry present in symmetric double well systems. The eigenfunctions of the system Hamiltonian are pairs of symmetric and antisymmetric states. Each eigenfunction $\psi$ can be decomposed in two terms dominantly localized on the "left" and "right" sides of a dividing surface which symmetrically splits the double well system,

$$
\psi=\psi_{1}+\psi_{\mathrm{r}} .
$$

$\psi_{1}$ and $\psi_{\mathrm{r}}$ denote the left and right parts of the wave function which are mirror images of each other,

$$
\psi_{\mathrm{r}}= \pm \hat{R} \psi_{1}
$$

Here $\hat{R}$ denotes the operator corresponding to a reflection with respect to the dividing surface. The splitting of $\psi$ into $\psi_{1}$ and $\psi_{\mathrm{r}}$ is based on an intuitive physical picture. Different detailed definitions could be used. A definition resulting in separation into orthogonal components can be achieved by introducing a function $h$ which acts as projection operator: $h$ equals unity on the right side of the dividing surface and vanishes on the left one. Then the $\psi_{1}$ and $\psi_{\mathrm{r}}$ would be defined as

$$
\begin{aligned}
& \psi_{\mathrm{r}}=h \psi, \\
& \psi_{1}=(1-h) \psi .
\end{aligned}
$$

An approximate or complete separation of the wave function $\psi$ into two localized components greatly simplifies the scaling problem discussed above. Consider a function $\psi_{\mathrm{r}}$ which is localized in the right well of Fig. 1. The minimal SPF basis required to qualitatively describe this function would require only one SPF per coordinate. Thus, the fundamental scaling problem discussed above for the representation of the delocalized wave function $\psi$ can be avoided if only the representation of localized wave functions $\psi_{1}$ or $\psi_{\mathrm{r}}$ within the MCTDH approach is required. The modifications required to utilize the symmetry considered above in the two methods described in Sec. II are discussed in Secs. III B-III D.

\section{B. Symmetry-adapted iterative diagonalization}

In the iterative Lanczos-type diagonalization approach that has been used in the previous communication, ${ }^{27}$ the recursion relation is modified to exploit the symmetry. Starting from an arbitrary wave-function $\left|\psi_{0}\right\rangle$ dominantly localized in the right well and its mirror image $\left|\psi_{1}\right\rangle=\hat{R}\left|\psi_{0}\right\rangle$, the modified Lanczos-type recursion relations used to build the diagonalization subspace read

$$
\begin{aligned}
& \left|\psi_{2 m}\right\rangle=C\left(\mathrm{e}^{-\beta \hat{H}} h\left|\psi_{2 m-2}\right\rangle-\sum_{i=0}^{2 m-1} \hat{P}\left|\psi_{i}\right\rangle \alpha_{i}\right), \\
& \left|\psi_{2 m+1}\right\rangle=\hat{R}\left|\psi_{2 m}\right\rangle,
\end{aligned}
$$

where the reflection operator $\hat{R}$ and the Heaviside function $h$ have been defined in Sec. III A. Within this modified procedure, one (modified) Lanczos-type iteration produces approximations to two eigenfunctions and associated eigenvalues. Because the functions $\psi_{2 m}$ and $\psi_{2 m+1}$ are nonorthogonal, a general eigenvalue solver is used for the diagonalization step.

Adding the projection operator $h$ before the imaginary time propagation enforces the propagated wavepacket $\mathrm{e}^{-\beta \hat{H}} h \psi$ to be mainly localized in one well only. Thus, $\psi_{2 m}$ is dominantly localized in the right well. Consequently, the set of wave functions $\left\{\psi_{0}, \psi_{2}, \psi_{4}, \ldots\right\}$ generates a basis to represent a wave function $\psi_{\mathrm{r}}$ mainly localized on the right side of a dividing surface. $\psi_{2 m+1}$ is the mirror image of $\psi_{2 m}$ and thus dominantly localized in the left well. Therefore the set of wave functions $\left\{\psi_{1}, \psi_{3}, \psi_{5}, \ldots\right\}$ generates a basis to represent a wave function $\psi_{1}$ mainly localized on the left side of a dividing surface. It should be noted that the wave functions of the mirror images $\psi_{2 m+1}$ do not need to be calculated or stored explicitly, but can always be computed "on the fly" by 
acting with the reflection operator $\hat{R}$ on the $\psi_{2 m}$. Consequently only wave functions of the $\psi_{\mathrm{r}}$-type have to be explicitly represented and an efficient MCTDH description can be achieved.

\section{Flux operator and symmetry-adapted Hamiltonians}

Section III B explained how the symmetry of the double well system has been exploited in the calculation presented in the previous communication. ${ }^{27}$ In the calculations presented here an improved scheme has been employed. First, block relaxation of state-averaged MCTDH wave functions (as described in Sec. II C) has been used instead of the Lanczos-type iterative diagonalization. This change facilitated the calculation of tunneling splittings of vibrationally excited states which could not be achieved previously. Second, the simple scheme to utilize the symmetry described above (Sec. III B) would not work as well in this new methodological context. A more robust scheme to exploit the symmetry has therefore been developed and is presented in the following.

To develop an efficient approach which utilizes symmetry considerations in the state-averaged MCTDH scheme of Sec. II C, it is helpful to first revisit the representation of the Hamiltonian operator employed. In Sec. III A, the eigenstates of the Hamiltonian in symmetric double well systems have been expressed as a sum of two symmetry related parts, see Eqs. (7) and (6). In a similar way the Hamiltonian can be decomposed,

$$
\begin{aligned}
\hat{H} & =h \hat{H} h+h \hat{H}(1-h)+(1-h) H(1-h)+(1-h) H h \\
& =h \hat{H} h+i h \hat{F}+(1-h) \hat{H}(1-h)-i(1-h) \hat{F},
\end{aligned}
$$

where $\hat{F}=i[\hat{H}, h]$ is the flux operator. Multiplying Eq. (9) from the left with $h$ and using the identity $h \hat{F}=h \hat{F}(1-h)$ one obtains

$$
h \hat{H} \psi=h \hat{H} h \psi+i h \hat{F}(1-h) \psi .
$$

Considering wave functions $\psi$ which are either symmetric or antisymmetric and are denoted as $\psi=\psi_{+}$or $\psi=\psi_{-}$, respectively, Eq. (6) implies $\hat{R} h \psi_{+}=(1-h) \psi_{+}$and $\hat{R} h \psi_{-}$ $=-(1-h) \psi_{-}$. For these wave functions the above equation can be rewritten as

$$
\begin{aligned}
h \hat{H} \psi_{+} & =h \hat{H} h \psi_{+}+i h \hat{F} \hat{R} h \psi_{+}, \\
h \hat{H} \psi_{-} & =h \hat{H} h \psi_{-}-i h \hat{F} \hat{R} h \psi_{-} .
\end{aligned}
$$

These equations motivate the definition of the new Hamiltonian operators $\hat{H}_{+}$and $\hat{H}_{-}$,

$$
\begin{aligned}
& \hat{H}_{+}:=h \hat{H} h+i h \hat{F} \hat{R} h, \\
& \hat{H}_{-}:=h \hat{H} h-i h \hat{F} \hat{R} h .
\end{aligned}
$$

The eigenstates of these new Hamiltonians are the projections $h \psi_{+}$and $h \psi_{-}$of the eigenstates $\psi_{+}$and $\psi_{-}$of the full Hamiltonian $\hat{H}$, respectively,

$$
\begin{aligned}
& \hat{H}_{+} h \psi_{+}=\hat{H}_{+} \psi_{+}=h \hat{H} \psi_{+}=E_{+} h \psi_{+}, \\
& \hat{H}_{-} h \psi_{-}=\hat{H}_{-} \psi_{-}=h \hat{H} \psi_{-}=E_{-} h \psi_{-} .
\end{aligned}
$$

Here $E_{+}$and $E_{-}$denote the corresponding eigenenergies of the states $\psi_{+}$and $\psi_{-}$, respectively. Since the wave functions $h \psi_{+}$and $h \psi_{-}$are localized on the right side of the dividing surface, they can be efficiently represented within the MCTDH approach and calculating the eigenstates of $\hat{H}_{+}$and $\hat{H}_{-}$avoids the scaling problems discussed in Sec. III A.

\section{A state-averaged MCTDH approach for double well problems}

Based on the $\hat{H}_{+}$and $\hat{H}_{-}$operators introduced in Sec. III C, one could straightforwardly compute the symmetric and antisymmetric eigenstates separately. However, if the calculation of tunneling splittings is the major aim, the simultaneous calculation of symmetric and antisymmetric states using a common basis of SPFs is preferable since it offers a balanced description of symmetric and antisymmetric states. Consequently one can expect that such a treatment shows a favorable cancellation of errors when computing the tunneling splittings.

To facilitate the simultaneous calculation of symmetric and antisymmetric eigenstates, a new Hamiltonian operator is introduced. This Hamiltonian depends on an additional degree of freedom $Q_{f+1}$ which can only take two discrete values. This additional discrete degree of freedom formally takes the same role as an electronic state index in a quantum dynamics calculation on two vibronically coupled PESs. Here, however, it will be used to denote whether a wave function is either symmetric or antisymmetric. Using a matrix-vector representation to specify the dependence on this additional degree of freedom, symmetric and antisymmetric wave functions are written as

$$
\psi_{+}=\left(\begin{array}{c}
\psi\left(Q_{1}, Q_{2}, \ldots, Q_{\mathrm{f}}, 1, t\right) \\
0
\end{array}\right)
$$

and

$$
\psi_{-}=\left(\begin{array}{c}
0 \\
\psi\left(Q_{1}, Q_{2}, \ldots, Q_{\mathrm{f}}, 2, t\right)
\end{array}\right) .
$$

Consequently the complete Hamiltonian reads in this notation as

$$
\hat{H}=\left(\begin{array}{cc}
\hat{H}_{+} & 0 \\
0 & \hat{H}_{-}
\end{array}\right) .
$$

It should be noted that this Hamiltonian is diagonal since the symmetric and antisymmetric wave-function subspaces are uncoupled.

The Hamiltonian is then employed in the state-averaged MCTDH calculations as described in Sec. II C. To guarantee a balanced description of symmetric and antisymmetric eigenstates, identical numbers of wave functions in the symmetric and antisymmetric subspaces are used. 
TABLE I. Grid sizes $N_{i}$ of DVR and FFT representations, numbers of SPFs $n_{i}$ employed in the reference and production MCTDH bases, normal mode frequencies, and a short description of the dominant type of motion for all coordinates (OOP and IP denote out-of-plane and in-plane motions; symmetry labels with respect to the $C_{2 v}$ symmetry of the transition state are given).

\begin{tabular}{|c|c|c|c|c|c|}
\hline \multirow[b]{2}{*}{$\tilde{Q}_{i}$} & \multirow[b]{2}{*}{$N_{i}$} & \multicolumn{2}{|c|}{$n_{i}$} & \multirow{2}{*}{$\begin{array}{l}\text { Frequency } \\
\qquad\left(\mathrm{cm}^{-1}\right)\end{array}$} & \multirow[b]{2}{*}{ Type of motion } \\
\hline & & Reference & Production & & \\
\hline 1 & 12 & 1 & 1 & 3344 & $\beta$-HC stretch $\left(\mathrm{A}_{1}\right)$ \\
\hline 2 & 12 & 1 & 2 & 3196 & $\alpha$-HC stretch $\left(\mathrm{A}_{1}\right)$ \\
\hline 3 & 12 & 1 & 2 & 3196 & $\alpha$-HC stretch $\left(\mathrm{B}_{2}\right)$ \\
\hline 4 & 16 & 2 & 2 & 1907 & Transfer $\mathrm{H}$ bend IP $\left(\mathrm{A}_{1}\right)$ \\
\hline 5 & 16 & 2 & 2 & 1724 & Ring deformation + transfer $\mathrm{H}$ IP $\left(\mathrm{B}_{2}\right)$ \\
\hline 6 & 12 & 1 & 1 & 1698 & CO stretch IP $\left(A_{1}\right)$ \\
\hline 7 & 12 & 1 & 1 & 1544 & Ring deformation $+\beta$-HC IP bend $\left(\mathrm{B}_{2}\right)$ \\
\hline 8 & 12 & 1 & 1 & 1408 & $\alpha$-HC IP bend $\left(\mathrm{A}_{1}\right)$ \\
\hline 9 & 12 & 1 & 1 & 1374 & $\alpha$-HC IP bend $\left(\mathrm{B}_{2}\right)$ \\
\hline 10 & 18 & 3 & 4 & 1314 & Transfer H OOP bend $\left(\mathrm{B}_{1}\right)$ \\
\hline 11 & 12 & 1 & 1 & 1139 & $\beta$-HC IP bend $\left(\mathrm{B}_{2}\right)$ \\
\hline 12 & 12 & 1 & 1 & 1097 & CC stretch $\left(A_{1}\right)$ \\
\hline 13 & 16 & 2 & 2 & 1058 & $\alpha$-HC symmetric OOP bend $\left(\mathrm{A}_{2}\right)$ \\
\hline 14 & 16 & 2 & 2 & 968 & $\alpha$-HC symmetric OOP bend $\left(\mathrm{B}_{1}\right)$ \\
\hline 15 & 18 & 1 & 2 & 953 & Ring deformation $\left(\mathrm{A}_{1}\right)$ \\
\hline 16 & 16 & 2 & 2 & 778 & $\beta$-HC OOP bend $\left(\mathrm{B}_{1}\right)$ \\
\hline 17 & 24 & 4 & 5 & 629 & Ring deformation $\left(\mathrm{A}_{1}\right)$ \\
\hline 18 & 16 & 3 & 3 & 576 & Ring deformation $\left(B_{2}\right)$ \\
\hline 19 & 24 & 4 & 4 & 384 & Antisymmetric $\alpha$ - $\mathrm{HC} / \mathrm{CO}$ OOP bend $\left(\mathrm{A}_{2}\right)$ \\
\hline 20 & 48 & 4 & 4 & 356 & $\alpha$-HC symmetric OOP bend $\left(\mathrm{B}_{1}\right)$ \\
\hline 21 & 64 & 5 & 6 & $1284 i$ & $\mathrm{H}$ transfer IP $\left(\mathrm{B}_{2}\right)$ \\
\hline
\end{tabular}

\section{SYSTEM DESCRIPTION AND NUMERICAL DETAILS}

Transition state normal mode coordinates $\mathbf{Q}$ are used to describe the malonaldehyde molecule. The normal mode coordinates and their respective frequencies are given in Table I. These coordinates are properly adapted to the symmetry of the double well system. Each coordinate can be characterized as being symmetric or antisymmetric with respect to the reflection, facilitating an easy implementation of the reflection operator.

To further reduce the correlation between the reaction coordinate and the other coordinates, a slightly transformed set of coordinates $\tilde{\mathbf{Q}}$ is employed in dynamics calculations,

$$
\begin{aligned}
& \tilde{Q}_{21}=Q_{21}, \\
& \tilde{Q}_{j}=Q_{j}-F_{j}\left(Q_{21}\right), \quad j=1, \ldots, 20,
\end{aligned}
$$

where the functions $F_{j}\left(Q_{21}\right)$ are defined as

$$
\begin{aligned}
& F_{j}\left(Q_{21}\right)=\frac{\sqrt{Q_{21}^{2}+\epsilon}}{Q_{21}^{(\mathrm{eq})}} Q_{j}^{(\mathrm{eq})}, \text { for symmetric } Q_{j}, \\
& F_{j}\left(Q_{21}\right)=\frac{Q_{21}}{Q_{21}^{(\mathrm{eq})}} Q_{j}^{(\mathrm{eq})}, \quad \text { for antisymmetric } Q_{j} .
\end{aligned}
$$

Here $Q_{j}^{(\text {eq })}$ denotes the equilibrium value in mode $Q_{j}$, for which one of the two possible minima has to be chosen. $\epsilon$ is a regularization parameter, guaranteeing a smooth shape of the $F_{j}$ functions. With this choice of $F_{j}$ the coordinates
$\widetilde{Q}_{1}, \ldots, \widetilde{Q}_{20}$ approximately vanish along the reaction path of the proton transfer.

Neglecting the vibrational angular momenta (see comment and references of our previous work ${ }^{27,28}$ and the specific results for malonaldehyde presented in Ref. 1 for the adequateness of this approximation), the kinetic energy operator in transition state normal mode coordinates reads

$$
\hat{T}=-\frac{1}{2} \sum_{i=1}^{21} \frac{\partial^{2}}{\partial Q_{i}^{2}} \text {. }
$$

Transforming the coordinate system according to Eq. (16) then yields the kinetic energy operator

$$
\begin{aligned}
\hat{T}= & -\frac{1}{2}\left(\sum_{i=1}^{21} \frac{\partial^{2}}{\partial \tilde{Q}_{i}^{2}}+\sum_{i, j=1}^{20} F_{i}^{\prime}\left(\widetilde{Q}_{21}\right) F_{j}^{\prime}\left(\widetilde{Q}_{21}\right) \frac{\partial^{2}}{\partial \widetilde{Q}_{i} \partial \widetilde{Q}_{j}}\right. \\
& \left.-\sum_{j=1}^{20}\left(F_{j}^{\prime}\left(\tilde{Q}_{21}\right) \frac{\partial}{\partial \tilde{Q}_{21}}+\frac{\partial}{\partial \tilde{Q}_{21}} F_{j}^{\prime}\left(\tilde{Q}_{21}\right)\right) \frac{\partial}{\partial \tilde{Q}_{j}}\right),
\end{aligned}
$$

with $F_{j}^{\prime}\left(Q_{21}\right)=d F_{j} / d Q_{21}$. (Please note that the corresponding formula in our communication ${ }^{27}$ included a typo). This kinetic energy operator is used in the present quantum dynamics calculations. Note that no change in the volume element results from transformation (16).

The PES of Yagi et $a l^{24}$ is employed. To reduce the numerical effort of the calculations, a modified implementation of the Shepard interpolation employed by the PES has been used. The changes have been confirmed to not alter any computed potential energy values relevantly and are described in detail in the Appendix. 


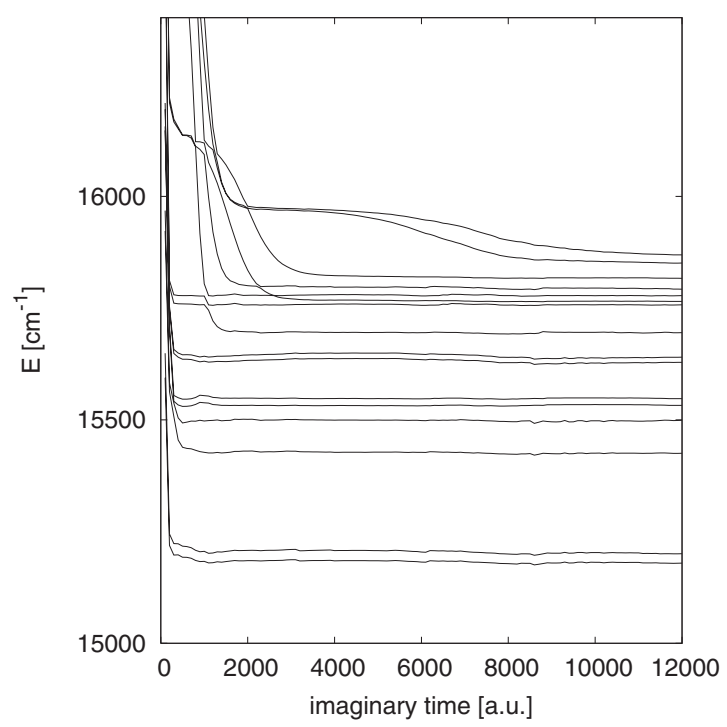

FIG. 2. Vibrational energy levels as a function of the total imaginary propagation time. 16 wavepackets and an iteration step length of $\beta=100$ a.u. have been used in the calculation.

Vibrational states have been calculated using the procedure described in Sec. III D. Imaginary time steps of $\beta$ $=100$ a.u. have been employed. The SPFs are represented numerically using a FFT scheme in coordinate $\widetilde{Q}_{21}$ and Hermite DVR schemes for the remaining modes. The grid sizes are shown in Table I. For the integration of the MCTDH equations of motion, a constant mean field (CMF) integration scheme $^{46}$ is used. Specifically, the CMF2 scheme of Ref. 47 is applied here. The CDVR approach ${ }^{43}$ is used to evaluate the potential energy matrix elements.

\section{RESULTS}

First, basic features of the calculations should be outlined. The schemes presented in Secs. II C and III D are based on iteration steps which consist of an imaginary time propagation for a time interval $\Delta t=i \beta$ followed by a subsequent Gram-Schmidt orthonormalization of the resulting set of wavepackets. The initial set of wavepackets at the start of the iteration sequence chosen in the present calculations consisted either on a guess based on a single harmonic oscillator model or, if available, the result of previous calculations using a small SPF basis. A typical convergence plot for a set of 16 simultaneous wavepackets based on an initial harmonic oscillator guess is shown in Fig. 2. There the convergence of energy versus the total propagation time is plotted, thus, the number of iterations is given by this total propagation time divided by $\beta$.

As can be seen in the figure, convergence occurs from bottom to top, i.e., the low lying eigenstates converge first and the higher ones last. This type of convergence is typical. The figure also shows that only at the beginning of the propagation the energy levels occur in pairs separated by relatively small energy differences. At later times, assigning pairs of states split by tunneling just based on energies becomes problematic. However, to calculate tunneling splittings, corresponding symmetric and antisymmetric states have to be identified. For this purpose, the wave functions of the calculated states had to be analyzed to identify the type of vibrational excitation. In the calculations presented below, for each type of excitation a pair consisting of symmetric and antisymmetric states could be uniquely identified, and the corresponding tunneling splitting could be calculated.

In order to obtain accurate results for tunneling splittings of vibrational states, the underlying SPF basis had to be converged. Due to the numerical effort, the SPF basis size had to be carefully adjusted and could not be arbitrarily enlarged. The convergence tests proceeded in two steps. First, a reference basis had to be determined, which allows a qualitatively correct description of the states under consideration.

In determining a suitable reference basis, two points are important. First, the number and type of excited states to be calculated has to be determined. To a first approximation, each excitation in a particular mode requires an additional SPF in that mode. In this study, the lowest six (split) excited vibrational states of malonaldehyde are investigated in detail. Through test calculations it is found that the lowest six pairs of states correspond to single excitations in modes $\widetilde{Q}_{17}, \widetilde{Q}_{18}$, $\widetilde{Q}_{19}$, and $\widetilde{Q}_{20}$, a simultaneous excitation in modes $\widetilde{Q}_{17}$ and $\widetilde{Q}_{19}$, and to a double excitation in $\widetilde{Q}_{17}$. An additional pair of states, corresponding to a double excitation in mode $\widetilde{Q}_{19}$, has been included in the calculations performed. However, the tunneling splitting of this seventh pair was found to be quite small (in the range of $5 \mathrm{~cm}^{-1}$ ) and could not be converged with suitable accuracy. We therefore decided to not report detailed results for this pair of states. The second point to consider is that the proton tunneling motion is a truly multidimensional process. Besides the proton transfer coordinate $Q_{21}$ a number of other modes also contribute strongly to the tunneling motion and thus have to be described particularly accurate. The normal modes contributing most strongly to the ground state splitting have also been determined in test calculations and have already been described in the previous communication. ${ }^{27}$ These include the in-plane modes $\widetilde{Q}_{4}, \widetilde{Q}_{5}$, $\widetilde{Q}_{17}$, and $\widetilde{Q}_{21}$, and the out-of-plane modes $\widetilde{Q}_{10}, \widetilde{Q}_{13}, \widetilde{Q}_{14}, \widetilde{Q}_{16}$, $\widetilde{Q}_{19}$, and $\widetilde{Q}_{20}$. Based on these considerations and tests, the reference basis has been chosen as $21^{5} 20^{4} 19^{4} 18^{3} 17^{4} 16^{2} 14^{2} 13^{2} 10^{3} 5^{2} 4^{2}$. Here the notation $\Pi_{i} i^{n_{i}}$ is used to denote all modes $\widetilde{Q}_{i}$ which are described using $n_{i}$ $>1$ SPFs. Modes $\widetilde{Q}_{j}$ described by a Hartree approximation, i.e., $n_{j}=1$, are not explicitly listed.

Vibrational energy levels calculated within this basis are given in Table II. A schematic representation of the levels is shown in Fig. 3. The excited states obtained include single and double excitations in modes $\widetilde{Q}_{17}$ and a single excitation in $\widetilde{Q}_{19}$, corresponding to a symmetric ring deformation and an antisymmetric out-of-plane $\mathrm{CH}$ bending motion, respectively. Single excitations are found in modes $\widetilde{Q}_{20}$ and $\widetilde{Q}_{18}$, corresponding to a symmetric out-of-plane bend and an inplane ring deformation, respectively. A combined excitation of modes $\widetilde{Q}_{17}$ and $\widetilde{Q}_{19}$ is also found. See Fig. 4 for representations of the corresponding normal mode displacements. Two immediate conclusions can be drawn from these results. First, as can be seen in Fig. 3, the tunneling splittings depend 
TABLE II. ZPE and vibrational excitation energies (in units of $\mathrm{cm}^{-1}$ ) of malonaldehyde. Results for the reference and production SPF bases (see Table I) are shown.

\begin{tabular}{cccccc}
\hline \hline & \multicolumn{2}{c}{ Reference basis } & & \multicolumn{2}{c}{ Production basis } \\
\cline { 2 - 3 } \cline { 5 - 6 } Vibrational level & Energy & Splitting & & Energy & Splitting \\
\hline $0_{+}$ & 15210 & & & 15185 & \\
$0_{-}$ & 23 & 23.0 & & 23 & 23.3 \\
$17_{+}^{1}$ & 240 & & & 196 & \\
$17_{-}^{1}$ & 302 & 61.3 & & 258 & 62.1 \\
$19_{+}^{1}$ & 311 & & & 302 & \\
$19_{-}^{1}$ & 326 & 14.7 & & 314 & 12.4 \\
$20_{+}^{1}$ & 421 & & & 415 & \\
$20_{-}^{1}$ & 430 & 9.2 & & 421 & 6.0 \\
$17_{+}^{2}$ & 503 & & & 414 & \\
$17_{-}^{2}$ & 607 & 104.0 & & 514 & 99.2 \\
$18_{+}^{1}$ & 550 & & & 553 & \\
$18_{-}^{1}$ & 568 & 17.9 & & 570 & 16.9 \\
$\left(19^{1} 17^{1}\right)_{+}$ & 555 & & & 506 & \\
$\left(19^{1} 17^{1}\right)_{-}$ & 599 & 43.8 & & 545 & 38.9 \\
\hline \hline
\end{tabular}

significantly on the type of vibrational excitation. The large differences in the splittings are remarkable. Second, with rising energy the labeling of the levels becomes less obvious. This is due to the rapidly increasing density of states and the strong dependence of the splitting on the type of vibrational excitation.

In the second step, additional convergence tests extending the reference basis have been performed. In these tests, the SPF basis is kept unchanged in all degrees of freedom except in a single mode or a single group of modes. Several modes have to be considered as groups since the different modes in the group are highly correlated. The basis size in each particular mode or group of modes is then increased until convergence is reached.

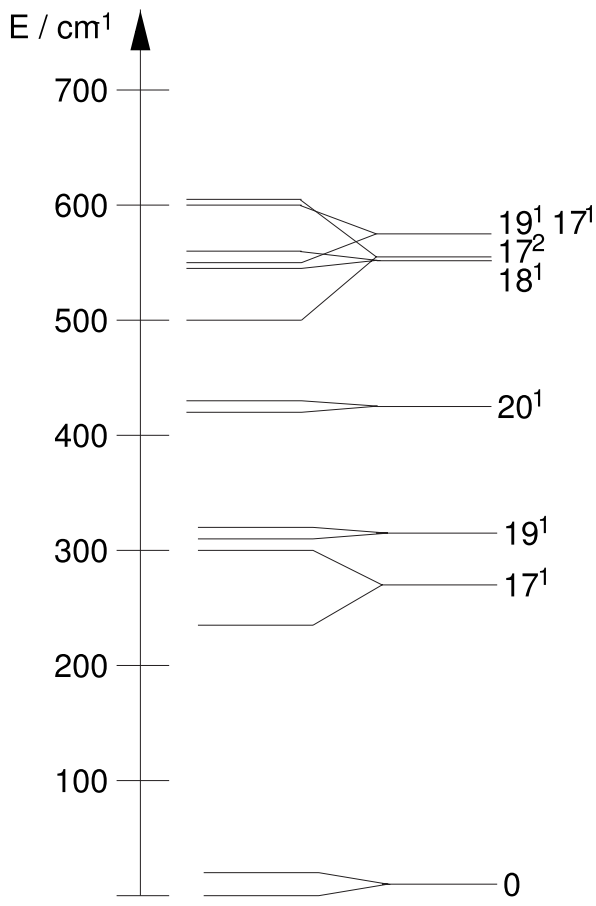

FIG. 3. Vibrational energy levels of malonaldehyde calculated with the reference SPF basis.
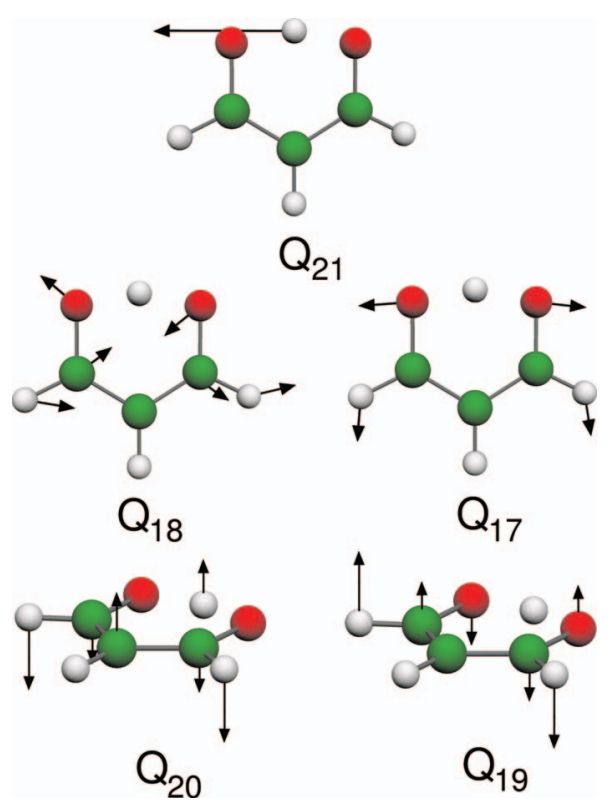

FIG. 4. Selected transition state normal modes in malonaldehyde: the hydrogen transfer mode $\widetilde{Q}_{21}$, the in-plane modes $\widetilde{Q}_{18}$ and $\widetilde{Q}_{17}$, and the out-ofplane modes $\widetilde{Q}_{20}$ and $\widetilde{Q}_{19}$.

Tables III and IV show the convergence of the tunneling splittings with the SPF basis size. In addition to the tunneling splittings and the zero point energy (ZPE), the lowest natural population of the SPFs in the mode under consideration is given. The natural populations ${ }^{31}$ of the SPFs in a particular mode give an indication about the quality of the wavefunction representation in that mode. For the groups of modes, the largest value of the lowest natural populations of the different modes in the group is given.

Considering the series in the five lowest frequency modes given in Table III, most of the tunneling splittings have been converged with an accuracy of approximately $1 \%-2 \%$. An exception is found in mode $\widetilde{Q}_{20}$, where the splittings in the excited states $17^{1}, 17^{1} 19^{1}$, and $17^{2}$ do not converge as smoothly as in the remaining modes. Increasing the SPF basis size in this mode beyond six SPFs gives no reliable results due to strong numerical noise in the iteration procedure. Thus, a relatively large contribution of $5 \%-7 \%$ to the overall error remains in the states with excitation in $\widetilde{Q}_{17}$.

The results for the higher frequency modes are shown in Table IV. The out-of-plane modes $\widetilde{Q}_{10}, \widetilde{Q}_{13}, \widetilde{Q}_{14}, \widetilde{Q}_{16}$ and the in-plane modes $\widetilde{Q}_{4}$ and $\widetilde{Q}_{5}$ can be seen to correlate most strongly with the reaction coordinate $\widetilde{Q}_{21}$. In these modes the Hartree approximation yields significant errors already for the ground state tunneling splitting. Additional SPFs for these modes have already been included in the reference basis. The inspection of the excited state tunneling splitting shows that additional SPFs are also required in modes $\widetilde{Q}_{2}$, $\widetilde{Q}_{3}, \widetilde{Q}_{10}$, and $\widetilde{Q}_{15}$. These have been included in the production basis. The convergence series in modes $\widetilde{Q}_{2}$ and $\widetilde{Q}_{3}$ is based on a slightly enlarged reference basis. Within this series an additional SPF has been added to the reference basis in $\widetilde{Q}_{15}$. Using the unmodified reference basis, a Fermi reso- 
TABLE III. Tunneling splittings and zero point energies (in units of $\mathrm{cm}^{-1}$ ) of malonaldehyde. The reference basis (see Table I) has been used, with varying numbers of SPFs in modes $\widetilde{Q}_{i}$ (the lines where $n_{i}$ equals the value taken in the production basis are shown in bold face). The rightmost column shows the lowest natural SPF population in the corresponding mode.

\begin{tabular}{|c|c|c|c|c|c|c|c|c|c|c|}
\hline Mode $i$ & $n_{i}$ & $\Delta_{0}$ & $\Delta_{17^{1}}$ & $\Delta_{19^{1}}$ & $\Delta_{20^{1}}$ & $\Delta_{18^{1}}$ & $\Delta_{19^{1} 17^{1}}$ & $\Delta_{17^{2}}$ & $\mathrm{ZPE}$ & SPF population \\
\hline \multirow{4}{*}{$\tilde{Q}_{21}$} & 4 & 23.9 & 73.3 & 15.2 & 10.6 & 17.9 & 53.0 & 130.0 & 15214 & $5 \times 10^{-3}$ \\
\hline & 5 & 23.0 & 61.3 & 14.6 & 9.2 & 17.8 & 43.4 & 103.2 & 15210 & $8 \times 10^{-4}$ \\
\hline & 6 & 23.1 & 63.4 & 14.4 & 9.4 & 17.6 & 45.0 & 103.4 & 15214 & $2 \times 10^{-4}$ \\
\hline & 7 & 23.3 & 63.2 & 14.7 & 9.5 & 17.3 & 45.1 & 103.3 & 15212 & $6 \times 10^{-5}$ \\
\hline \multirow{4}{*}{$\widetilde{Q}_{20}$} & 2 & 22.3 & 65.1 & 15.4 & 10.0 & 16.6 & 51.1 & 110.1 & 15213 & $1 \times 10^{-1}$ \\
\hline & 3 & 21.8 & 64.4 & 15.2 & 5.5 & 17.4 & 46.8 & 116.3 & 15203 & $5 \times 10^{-3}$ \\
\hline & 4 & 23.0 & 61.3 & 14.7 & 9.2 & 17.9 & 43.8 & 104.0 & 15210 & $9 \times 10^{-4}$ \\
\hline & 5 & 22.8 & 65.1 & 14.9 & 9.3 & 17.7 & 46.5 & 111.3 & 15209 & $5 \times 10^{-4}$ \\
\hline \multirow[t]{3}{*}{$\widetilde{Q}_{19}$} & 3 & 24.1 & 60.5 & 14.2 & 9.5 & 18.5 & 43.5 & 104.5 & 15211 & $9 \times 10^{-2}$ \\
\hline & 4 & 23.0 & 61.3 & 14.7 & 9.2 & 17.9 & 43.8 & 104.0 & 15210 & $2 \times 10^{-3}$ \\
\hline & 5 & 23.3 & 60.9 & 14.6 & 9.4 & 18.0 & 42.3 & 102.3 & 15214 & $2 \times 10^{-4}$ \\
\hline \multirow[t]{3}{*}{$\widetilde{Q}_{18}$} & 2 & 23.3 & 60.7 & 14.8 & 9.3 & 18.3 & 43.5 & 103.5 & 15211 & $1 \times 10^{-1}$ \\
\hline & 3 & 23.0 & 61.3 & 14.7 & 9.2 & 17.9 & 43.8 & 104.0 & 15210 & $2 \times 10^{-3}$ \\
\hline & 4 & 22.9 & 60.9 & 14.6 & 9.1 & 17.4 & 43.2 & 103.6 & 15212 & $9 \times 10^{-5}$ \\
\hline \multirow[t]{4}{*}{$\widetilde{Q}_{17}$} & 3 & 23.6 & 63.8 & 14.7 & 9.4 & 18.0 & 48.1 & 131 & 15212 & $4 \times 10^{-2}$ \\
\hline & 4 & 23.0 & 61.3 & 14.7 & 9.2 & 17.9 & 43.8 & 104.0 & 15210 & $3 \times 10^{-3}$ \\
\hline & 5 & 23.8 & 63.8 & 14.8 & 9.5 & 18.0 & 45.5 & 102.1 & 15214 & $4 \times 10^{-4}$ \\
\hline & 6 & 23.7 & 63.6 & 14.9 & 9.5 & 18.2 & 45.2 & 102.1 & 15214 & $4 \times 10^{-6}$ \\
\hline
\end{tabular}

nance appears if more than one SPF in $\widetilde{Q}_{2}$ and $\widetilde{Q}_{3}$ is used. This Fermi resonance disappears again if the basis is increased in $\widetilde{Q}_{15}$. Thus, it is an artifact of an insufficiently sized SPF basis. To avoid unphysical behavior in the $\widetilde{Q}_{2}$ and $\widetilde{Q}_{3}$ convergence series, a larger SPF basis in $\widetilde{Q}_{15}$ had to be used here. The resulting number of SPFs used in the production calculation is summarized in Table I.

Based on the convergence studies for the different modes or groups of modes, the degree of convergence achieved in the final production calculation can be estimated. To this end, the accuracies of the tunneling splittings obtained in each of the convergence series are summarized in Table V. The table shows for each convergence series the differences between the tunneling splittings calculated with the largest SPF basis size and the size chosen for production. The differences in the individual series are typically only a few percent and the maximum errors seen for the tunneling splittings of the ground and vibrationally excited states, $\Delta_{0}, \Delta_{17^{1}}, \Delta_{19^{1}}, \Delta_{20^{1}}$, $\Delta_{18^{1}}, \Delta_{19^{1}{ }^{1} 7^{1}}$, and $\Delta_{17^{2}}$, are $3 \%, 6 \%, 7 \%, 15 \%, 4 \%, 7 \%$, and $7 \%$, respectively.

However, since malonaldehyde has 21 internal coordinates and 14 individual convergence series have been studied, a careful investigation of the total convergence error has to consider the question of error accumulation. Therefore the sum of the errors of all individual convergence series, $\Sigma_{i} \delta_{i}$, and the root of the sum of squares, $\sqrt{\Sigma_{i} \delta_{i}^{2}}$, are also given in Table V. These two numbers indicate how errors in the individual convergence series might accumulate. $\Sigma_{i} \delta_{i}$ gives an estimate for the improvement which would be achieved by a MCTDH calculation with one additional SPF in each coordinate. While $\Sigma_{i} \delta_{i}$ indicates whether errors obtained from the individual convergence series show a common direction, $\sqrt{\sum_{i} \delta_{i}^{2}}$ measures the error which would be expected if the individual convergence errors would be randomly oriented.

Since the $\Sigma_{i} \delta_{i}$ and $\sqrt{\Sigma_{i} \delta_{i}^{2}}$ for the tunneling splittings are of roughly comparable size, a strong common trend of all convergence errors in one direction does not seem to be present. The relative accuracies obtained for the larger tunneling splitting is higher than for the smaller ones: While a $10 \%$ accuracy estimate for the computed values of the larger tunneling splittings seems reasonable, the error estimate increases up to $20 \%$ for the value of the smallest tunneling splitting $\Delta_{20^{1}}$.

For the computed ZPE, almost all convergence errors of the individual convergence series point in the same direction: Increasing the SPF basis almost always decreases the ZPE. This is an obvious consequence of the variational nature of the MCTDH approach. Increasing the SPF basis improves the description of the ground state wave function and therefore results in a smaller ZPE value computed. While the inherent error compensation build into the theoretical scheme for computing the tunneling splitting facilitates more rapid convergence of the computed tunneling splittings with the SPF basis size, the computation of an accurate value of the ZPE requires a large SPF basis set until convergence to the same absolute accuracy is achieved. The similar tendency can also be seen when comparing the accuracy of the computed absolute vibrational excitation energies and tunneling splittings. In Table II, differences between results obtained with the reference SPF basis and the production SPF basis are significantly larger for the absolute energies than for the tunneling splittings. 
TABLE IV. Tunneling splittings and zero point energies (in units of $\mathrm{cm}^{-1}$ ) of malonaldehyde. The reference basis (see Table I) has been used, with varying numbers of SPFs in the different groups of modes (the lines where $n_{i}$ equals the value taken in the production basis are shown in bold face). The rightmost column shows the lowest natural SPF population.

\begin{tabular}{|c|c|c|c|c|c|c|c|c|c|c|}
\hline Mode $i$ & $n_{i}$ & $\Delta_{0}$ & $\Delta_{17^{1}}$ & $\Delta_{19^{1}}$ & $\Delta_{20^{1}}$ & $\Delta_{18^{1}}$ & $\Delta_{19^{1} 17^{1}}$ & $\Delta_{17^{2}}$ & ZPE & SPF population \\
\hline \multirow{3}{*}{$\tilde{Q}_{13}, \widetilde{Q}_{14}, \widetilde{Q}_{16}$} & 1 & 26.5 & 66.2 & 17.9 & 12.6 & 22.1 & 53.2 & 116.7 & 15208 & 1 \\
\hline & 2 & 23.0 & 61.3 & 14.7 & 9.2 & 17.9 & 43.8 & 104.0 & 15210 & $8 \times 10^{-3}$ \\
\hline & 3 & 23.6 & 61.9 & 14.4 & 9.5 & 17.8 & 44.1 & 107.0 & 15209 & $4 \times 10^{-4}$ \\
\hline \multirow{3}{*}{$\widetilde{Q}_{15}$} & 1 & 23.0 & 61.3 & 14.7 & 9.2 & 17.9 & 43.8 & 104.0 & 15210 & 1 \\
\hline & 2 & 22.6 & 58.5 & 13.6 & 8.2 & 16.0 & 41.2 & 99.2 & 15202 & $3 \times 10^{-2}$ \\
\hline & 3 & 22.5 & 57.8 & 13.6 & 8.1 & 16.1 & 40.7 & 98.3 & 15200 & $6 \times 10^{-4}$ \\
\hline \multirow{2}{*}{$\tilde{Q}_{11}, \tilde{Q}_{12}$} & 1 & 23.0 & 61.3 & 14.7 & 9.2 & 17.9 & 43.8 & 104.0 & 15210 & 1 \\
\hline & 2 & 22.9 & 61.3 & 14.4 & 8.9 & 17.3 & 43.3 & 104.9 & 15206 & $3 \times 10^{-3}$ \\
\hline \multirow{4}{*}{$\tilde{Q}_{10}$} & 2 & 20.7 & 60.5 & 13.8 & 9.0 & 15.8 & 42.7 & 104.7 & 15213 & $1 \times 10^{-2}$ \\
\hline & 3 & 23.0 & 61.3 & 14.7 & 9.2 & 17.9 & 43.8 & 104.0 & 15210 & $3 \times 10^{-3}$ \\
\hline & 4 & 22.0 & 62.2 & 14.8 & 9.4 & 17.0 & 45.8 & 108.2 & 15206 & $4 \times 10^{-4}$ \\
\hline & 5 & 21.8 & 61.4 & 14.3 & 9.4 & 16.6 & 44.9 & 108.4 & 15204 & $2 \times 10^{-4}$ \\
\hline \multirow{2}{*}{$\tilde{Q}_{8}, \tilde{Q}_{9}$} & 1 & 23.0 & 61.3 & 14.7 & 9.2 & 17.9 & 43.8 & 104.0 & 15210 & 1 \\
\hline & 2 & 23.8 & 60.6 & 13.8 & 10.1 & 17.9 & 40.9 & 101.1 & 15195 & $9 \times 10^{-3}$ \\
\hline \multirow{2}{*}{$\widetilde{Q}_{6}, \widetilde{Q}_{7}$} & 1 & 23.0 & 61.3 & 14.7 & 9.2 & 17.9 & 43.8 & 104.0 & 15210 & 1 \\
\hline & 2 & 23.0 & 61.1 & 14.5 & 8.7 & 17.4 & 42.6 & 106.0 & 15198 & $3 \times 10^{-3}$ \\
\hline \multirow{3}{*}{$\widetilde{Q}_{4}, \widetilde{Q}_{5}$} & 1 & 29.5 & 76.8 & 19.1 & 11.7 & 24.8 & 55.6 & 124.2 & 15275 & 1 \\
\hline & 2 & 23.0 & 61.3 & 14.7 & 9.2 & 17.9 & 43.8 & 104.0 & 15210 & $2 \times 10^{-2}$ \\
\hline & 3 & 23.6 & 63.7 & 14.5 & 9.0 & 18.0 & 45.4 & 107.8 & 15210 & $7 \times 10^{-4}$ \\
\hline \multirow{3}{*}{$\tilde{Q}_{2}, \widetilde{Q}_{3}{ }^{\mathrm{a}}$} & 1 & 22.6 & 58.5 & 13.6 & 8.2 & 16.0 & 41.2 & 99.2 & 15202 & 1 \\
\hline & 2 & 22.7 & 60.0 & 11.4 & 6.0 & 14.9 & 37.0 & 101.7 & 15188 & $4 \times 10^{-3}$ \\
\hline & 3 & 23.0 & 60.1 & 11.4 & 5.9 & 14.9 & 37.6 & 102.0 & 15189 & $7 \times 10^{-5}$ \\
\hline \multirow[t]{2}{*}{$\widetilde{Q}_{1}$} & 1 & 23.0 & 61.3 & 14.7 & 9.2 & 17.9 & 43.8 & 104.0 & 15210 & 1 \\
\hline & 2 & 23.1 & 61.9 & 14.4 & 8.9 & 17.7 & 43.2 & 103.3 & 15203 & $2 \times 10^{-3}$ \\
\hline
\end{tabular}

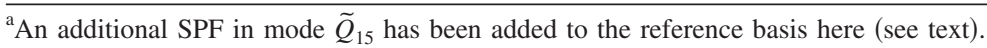

\section{DISCUSSION}

The results presented in Sec. V show that the size of the tunneling splitting strongly depends on the type of vibrational excitation. A simple semiclassical picture can be used to straightforwardly explain these findings. If an excited vibrational motion can result in a simultaneous shortening of both $\mathrm{O}-\mathrm{H}$ distances, then the effective barrier for tunneling is (temporarily) decreased and the tunneling is dynamically enhanced. An increase in the tunneling splitting upon excitation of this vibrational mode is the consequence. Oppositely, if an excited vibrational motion always increases both $\mathrm{O}-\mathrm{H}$ distances, the effective barrier for tunneling is only increased and a decrease in the tunneling splitting upon excitation follows.

As a basis for the following detailed discussion, the relevant transition state normal coordinates are displayed in Fig. 4. Now the detailed investigation can start with the lowest vibrationally excited pair of states. These states contain one quantum of excitation in normal mode $\widetilde{Q}_{17}$. As seen in Fig. 4, this mode corresponds to a symmetric ring opening/ closing. This vibrational motion modulates the $\mathrm{O}-\mathrm{O}$ distance and simultaneously decreases/increases both $\mathrm{O}-\mathrm{H}$ distances. It is therefore expected to significantly increase the tunneling splitting. This expectation is in perfect agreement with the computed results. A pronounced increase in the computed tunneling splitting, $\Delta_{17^{1}}=62.1 \mathrm{~cm}^{-1}$, compared to the ground state tunneling splitting, $\Delta_{0}=23.3 \mathrm{~cm}^{-1}$, is found. Moreover, a further increase in the tunneling splitting is found if a second quantum of excitation is set into this mode: $\Delta_{172}$ $=99.2 \mathrm{~cm}^{-1}$. Similarly, the tunneling splitting of the vibrational states with a combined excitation in modes $\widetilde{Q}_{17}$ and $\widetilde{Q}_{19}\left(\Delta_{17^{1} 19^{1}}=38.9 \mathrm{~cm}^{-1}\right)$ is significantly larger than the splitting of the vibrational states with excitation in mode $\widetilde{Q}_{19}$ only $\left(\Delta_{19^{1}}=12.4 \mathrm{~cm}^{-1}\right)$.

Beside the excitation in mode $\widetilde{Q}_{17}$, also vibrational excitations in modes $\widetilde{Q}_{18}, \widetilde{Q}_{19}$, and $\widetilde{Q}_{20}$ are found for the vibrationally excited states studied. None of these three vibrational modes can result in a significant or simultaneous shortening of both $\mathrm{O}-\mathrm{H}$ distances. The two out-of-plane modes $\widetilde{Q}_{19}$ and $\widetilde{Q}_{20}$ always increase both O-H distances, this effect being more pronounced in $\widetilde{Q}_{20}$ than in $\widetilde{Q}_{19}$. Conse- 
TABLE V. Accuracies of tunneling splitting values (in units $\mathrm{of} \mathrm{cm}^{-1}$ ). For each convergence series from Tables III and IV, the differences $\delta$ in tunneling splittings and the ZPE calculated with the two largest SPF basis are shown.

\begin{tabular}{|c|c|c|c|c|c|c|c|c|}
\hline Mode & $\delta\left(\Delta_{0}\right)$ & $\delta\left(\Delta_{171}\right)$ & $\delta\left(\Delta_{191}\right)$ & $\delta\left(\Delta_{20^{1}}\right)$ & $\delta\left(\Delta_{18^{1}}\right)$ & $\delta\left(\Delta_{19^{1}{ }_{17}}\right)$ & $\delta\left(\Delta_{17^{2}}\right)$ & ZPE \\
\hline$\tilde{Q}_{21}$ & -0.2 & -0.2 & -0.3 & 0.1 & -0.3 & 0.1 & -0.1 & 2 \\
\hline$\tilde{Q}_{20}$ & -0.2 & 3.8 & 0.2 & 0.1 & -0.2 & 2.7 & 7.3 & -1 \\
\hline$\widetilde{Q}_{19}$ & 0.3 & -0.4 & -0.1 & 0.2 & 0.1 & -1.5 & -1.7 & 4 \\
\hline$\tilde{Q}_{18}$ & -0.1 & -0.4 & -0.1 & -0.1 & -0.5 & -0.6 & -0.4 & 2 \\
\hline$\tilde{Q}_{17}$ & -0.1 & -0.2 & 0.1 & 0.0 & 0.2 & -0.3 & 0.0 & 0 \\
\hline$\widetilde{Q}_{13}, \widetilde{Q}_{14}, \widetilde{Q}_{16}$ & 0.6 & 0.6 & -0.3 & 0.3 & -0.1 & 0.3 & 3.0 & -1 \\
\hline$\tilde{Q}_{15}$ & -0.1 & -0.7 & 0.0 & -0.1 & 0.1 & -0.5 & -0.9 & -2 \\
\hline$\widetilde{Q}_{11}, \widetilde{Q}_{12}$ & -0.1 & 0.0 & -0.3 & -0.3 & -0.6 & -0.5 & 0.9 & -4 \\
\hline$\tilde{Q}_{10}$ & -0.2 & -0.8 & -0.5 & 0.0 & -0.4 & -0.9 & 0.2 & -2 \\
\hline$\widetilde{Q}_{8}, \widetilde{Q}_{9}$ & 0.8 & -0.7 & -0.9 & 0.9 & 0.0 & -2.9 & -2.9 & -15 \\
\hline$\tilde{Q}_{6}, \widetilde{Q}_{7}$ & 0.0 & -0.2 & -0.2 & -0.5 & -0.5 & -1.2 & 2.0 & -12 \\
\hline$\tilde{Q}_{4}, \widetilde{Q}_{5}$ & 0.6 & 2.4 & -0.2 & -0.2 & 0.1 & 1.6 & 3.8 & 0 \\
\hline$\tilde{Q}_{2}, \widetilde{Q}_{3}{ }^{\mathrm{a}}$ & 0.3 & 0.1 & 0.0 & -0.1 & 0.0 & 0.6 & 0.3 & 1 \\
\hline$\widetilde{Q}_{1}$ & 0.1 & 0.6 & -0.3 & -0.3 & -0.2 & -0.7 & -0.7 & -7 \\
\hline $\operatorname{Sum}^{\mathrm{b}} \Sigma_{i} \delta_{i}$ & 1.7 & 3.9 & -2.9 & 0.0 & -2.3 & -3.8 & 10.8 & -36 \\
\hline$\sqrt{\sum_{i} \delta_{i}^{2}}$ & 1.3 & 4.8 & 1.3 & 1.2 & 1.1 & 5.0 & 9.7 & 21.6 \\
\hline Present result & 23.3 & 62.1 & 12.4 & 6.0 & 16.9 & 38.9 & 99.2 & 15185 \\
\hline $\mathrm{DMC}^{\mathrm{c}}$ & 25.7 & & & & & & & 15122 \\
\hline
\end{tabular}

${ }^{a}$ An additional SPF in mode $\widetilde{Q}_{15}$ has been added to the reference basis here.

${ }^{\mathrm{b}}$ Where the sum index $i$ runs over all modes and groups of modes.

${ }^{\mathrm{c}}$ From Ref. 28.

quently, a decrease in the computed tunneling splitting can be observed for the vibrational states $19^{1}$ and $20^{1}$. The splitting $\Delta_{20^{1}}=6.0 \mathrm{~cm}^{-1}$ is even smaller than $\Delta_{19^{1}}=12.4$. The inplane mode $\widetilde{Q}_{18}$ results in an asynchronous change in the two $\mathrm{O}-\mathrm{H}$ distances with a seemingly slight net increase in the averaged $\mathrm{O}-\mathrm{H}$ distance. The computed slight decrease in the tunneling splitting, $\Delta_{18}=16.9 \mathrm{~cm}^{-1}$, compared to the ground state tunneling splitting, $\Delta_{0}=23.3 \mathrm{~cm}^{-1}$, fits well into this picture.

Some results of the present calculations can be compared to other theoretical and experimental results. As theoretical results for the same PES, ground state tunneling splittings of $25.7 \pm 0.3 \mathrm{~cm}^{-1}$ obtained by POITSE DMC calculations and of $25 \mathrm{~cm}^{-1} \pm 10 \%$ computed using the MCTDH method with the symmetry-adapted iterative diagonalization approach have been reported in our previous work. ${ }^{27,28}$ The present result, $23.3 \mathrm{~cm}^{-1}$ with an error estimate of about $10 \%$, agrees with the previous results within the assumed error margins. It could additionally be noted that the assumed improvement of the results upon further increase in the SPF basis as given in Table V, $1.7 \mathrm{~cm}^{-1}$, would set the present result even closer to the more accurate POITSE DMC result. The POITSE DMC calculations also yield an accurate value of the ZPE: ${ }^{.8} 15122 \pm 4 \mathrm{~cm}^{-1}$. The result obtained from our calculation with the largest (production) SPF basis is $15185 \mathrm{~cm}^{-1}$ and thus about $60 \mathrm{~cm}^{-1}$ too high. This finding is not surprising. The results shown in Table $\mathrm{V}$ indicated that the present production SPF basis size is not sufficient to converge the computed value of the ZPE beyond an error margin of several dozen wavenumbers: A decrease in the ZPE by $36 \mathrm{~cm}^{-1}$ was estimated as the effect of using one additional SPF in each coordinate. In conclusion, in the present state the MCTDH calculations cannot rival the accuracy obtained by the POITSE DMC calculations for the ground state tunneling splitting and the ZPE.

The computed excited state tunneling splittings, which cannot be obtained using the POITSE DMC technique, are therefore a particularly relevant result of the present work. Other theoretical work of reasonable predictive accuracy for these splittings was only published by Tew et al. ${ }^{33}$ Their most accurate calculation employed a VCI with up to quadruple excitations and a reaction surface PES including up to three body contributions. Their reaction surface PES and the PES of Yagi et al. ${ }^{24}$ used in the present work rely on ab initio data of the same level of accuracy. Thus, errors resulting from inaccuracies of the electronic structure calculation should be very similar in both works and differences should result only from differences in the PES construction schemes and the dynamical calculations. Discussing their approach, Tew et al. considered the computed ground state tunneling splitting, $15 \mathrm{~cm}^{-1}$, and one of the excited state tunneling splittings, $58 \mathrm{~cm}^{-1}$, reliable (the restrictions in the PES model did not allow to obtain reliable results for other excited state tunneling splittings). Using the notation of the present work, this excited state tunneling splitting corresponds to the $17^{1}$ excitation. The agreement with the present result, $\Delta_{171}=62.1 \mathrm{~cm}^{-1}$, is very good (considering the respective error margins of the two results).

Considering only the low lying vibrationally excited states investigated here, experimental results are only available for the $\Delta_{18^{1}}$ splitting. Analyzing their spectroscopic data, Seliskar and Hofmann ${ }^{8}$ found a decrease in this splitting of 
$6 \mathrm{~cm}^{-1}$ relative to the ground state splitting: $\Delta_{18^{1}}-\Delta_{0}$ $=-6 \mathrm{~cm}^{-1}$. This value is in very good agreement with the present theoretical result, $\Delta_{18^{1}}-\Delta_{0}=-6.4 \mathrm{~cm}^{-1}$. When comparing the present theoretical results with experiment, one should remember that the PES employed in the present work shows a somewhat too low barrier and consequently overestimates the ground state tunneling splitting by about $4 \mathrm{~cm}^{-1}$. However, the change in the tunneling splitting upon vibrational excitation mainly depends on the geometric character of the vibrational motion excited. It can therefore be expected to depend less sensitively on the barrier height than the absolute value of a specific tunneling splitting. Consequently, the good agreement between the present theoretical value and experiment does not appear to be fortuitous.

\section{CONCLUSIONS AND PERSPECTIVES}

Theoretical developments facilitating the efficient use of the MCTDH approach for the calculation of tunneling splittings in proton transfer systems have been presented and discussed. The schemes employed utilize the symmetry of the double well system. As a consequence, the wave function which has to be represented by the MCTDH scheme is localized mainly or even entirely in a single well only. This decreases the number of SPFs required to converge the MCTDH results and consequently reduces the numerical effort compared to a simple straightforward approach. Two different schemes are described: the symmetry-adapted iterative diagonalization approach utilized to compute the results previously communicated ${ }^{27}$ and a new, more efficient approach. In the new approach, two effective Hamiltonians are introduced. The eigenstates of these two Hamiltonians are located in only one of the two wells and are the projected components of the symmetric and antisymmetric eigenstates, respectively, of the full system Hamiltonian. The stateaveraged MCTDH approach can then be used to efficiently compute the tunneling splittings in a balanced way. Consequently, the approach can yield converged results for the tunneling splitting with smaller single-particle basis sets than would be required for the convergence of the corresponding absolute vibrational energies.

Using these techniques, rigorous full-dimensional quantum dynamics calculations of the tunneling splitting not only of the ground state but also of the low lying excited vibrational states of malonaldehyde could be presented. While for the ground state tunneling splitting results of superior accuracy can be obtained by POITSE DMC calculations, ${ }^{28}$ only the MCTDH approach can presently provide rigorous results for the tunneling splittings of vibrationally excited states. The present calculations still used the PES of Yagi et al. ${ }^{24}$ which is based on $a b$ initio data on MP2 level and underestimates the barrier height for proton transfer by about 0.4 $\mathrm{kcal} / \mathrm{mol}$ or $10 \%$. However, good agreement of the calculated change in the tunneling splitting upon vibrational excitation with experiment was found for the single case where experimental data are available. We hope that the present work will inspire experimental efforts to measure tunneling splittings for a larger set of vibrationally excited states.

Based on the results of the present work, the perspec- tives for future work seem to be obvious. Similar calculations should be repeated on the recently developed PES of Wang et al. ${ }^{1}$ which is significantly more accurate than the PES of Yagi et al. employed in the present work. However, very recent test calculations indicated that for the PES of Wang et al., the computation of the potential energy values is computationally significantly more demanding than for the PES of Yagi et al. Since the computational effort of our present MCTDH calculations (which use the CDVR scheme to evaluate the potential energy matrix elements) depends crucially on the speed of the subroutine providing the potential energy values at given geometries, a significantly increased numerical effort of such a study compared to the present one can be expected.

The present results show that the rigorous treatment of the 21-dimensional malonaldehyde system pushes the singlelayer MCTDH approach used here to its limits. A further increase in the SPF basis, which would have been desirable to facilitate the accurate calculation of absolute energies of the vibrational levels and to further increase the accuracy of the calculated tunneling splittings, was impossible due to the limits posted by our computational resources. Further increasing the efficiency of our theoretical and numerical approach thus is a key issue. Application and further development of the multilayer MCTDH approach ${ }^{48-50}$ offers an interesting perspective toward this aim. Thus, multilayer MCTDH calculations studying malonaldehyde will be a topic of our future research.

\section{ACKNOWLEDGMENTS}

Financial support by the Deutsche Forschungsgemeinschaft and the Fond der Chemischen Industrie is gratefully acknowledged.

\section{APPENDIX: EFFICIENT EVALUATION OF PESs BASED ON SHEPARD INTERPOLATION}

Employing modified Shepard interpolation, the potential $V(\mathbf{q})$ at a point $\mathbf{q}$ is given by the weighted sum of local estimates $T\left(\mathbf{q}, \mathbf{q}_{i}\right)$ from the different reference points $\mathbf{q}_{i}{ }^{25,51}$

$$
V(\mathbf{q})=\sum_{i} w_{i}(\mathbf{q}) T\left(\mathbf{q}, \mathbf{q}_{i}\right)
$$

The $w_{i}(\mathbf{q})$ are weighting functions. The value of $w_{i}(\mathbf{q})$ depends on the distance between the point $\mathbf{q}$ considered and the reference point $\mathbf{q}_{i}$ and is defined by some suitable measure of distance.

The numerical effort to evaluate the potential energy function (A1) obviously depends on the number of terms in the sum. Since typically only a small number of reference points $\mathbf{q}_{i}$ is sufficiently close to any point $\mathbf{q}$ considered, the weights $w_{i}(\mathbf{q})$ are very small for most $i$ 's. To speed up the numerical evaluation of the potential, these terms can be neglected in sum (A1) without changing the PES relevantly. Devising appropriate schemes to determine such terms is thus of crucial importance for the numerical efficiency of the PES evaluation.

An obvious approach, which has been implemented by Collins and co-workers, used a threshold value to neglect 
terms where $w_{i}(\mathbf{q})$ is sufficiently small. An additional criterion based on physical considerations that allows neglecting an even larger number of terms can be introduced. When computing the potential energy $V(\mathbf{q})$ at a point $\mathbf{q}$, the local estimate $T\left(\mathbf{q}, \mathbf{q}_{n}\right)$ from the reference point $\mathbf{q}_{n}$ closest to $\mathbf{q}$ [i.e., the reference point with the highest weight $\left.w_{n}(\mathbf{q})\right]$ defines the most reliable single point based estimate for $V(\mathbf{q})$. Local estimates $T\left(\mathbf{q}, \mathbf{q}_{i}\right)$ from other reference points $\mathbf{q}_{i}$ which differ strongly from $T\left(\mathbf{q}, \mathbf{q}_{n}\right)$ [or another good estimate for $V(\mathbf{q})]$ can be considered physically unreasonable. These reference points $\mathbf{q}_{i}$ are too distant from $\mathbf{q}$ to allow a reasonable estimate of the potential. It is therefore preferable to neglect the erroneous contributions from these points in sum (A1). The revised expression for the potential estimate,

$$
V_{\text {estimate }}(\mathbf{q})=\frac{\sum_{j} w_{j}(\mathbf{q}) T\left(\mathbf{q}, \mathbf{q}_{j}\right)}{\sum_{j} w_{j}(\mathbf{q})},
$$

includes only reasonable reference points $\mathbf{q}_{j}$ in the summation.

Different strategies can be used to implement this idea. In previous Shepard interpolated PESs constructed by our research group ${ }^{52,53}$ for a given $\mathbf{q}$ the weights $w_{i}(\mathbf{q})$ are sorted by size and summation (A2) proceeded summing points in order of decreasing weights $w_{j}(\mathbf{q})$. The summation was stopped once the next reference point $\mathbf{q}_{m}$ in the series would yield a physically unreasonable $T\left(\mathbf{q}, \mathbf{q}_{j}\right)$. In the present work (as well as our previous work in Refs. 27 and 28) a simplified (and therefore numerically even cheaper) procedure was employed. It utilized the energy at the reference point as an estimate for $T\left(\mathbf{q}, \mathbf{q}_{i}\right)$ and discarded reference points showing energy differences of more than $0.3 \mathrm{eV}$ relative to the nearmost reference point.

It was found that this procedure considerably increased the efficiency of the PES evaluation for malonaldehyde. Significant differences between the potential computed with or without this truncation were only observed in regions showing high potential energy values and a small density of reference points. In these regions the original PES values did not seem to be particularly accurate anyhow and the values obtained by the truncated sum even seemed to be physically more reasonable.

\footnotetext{
${ }^{1}$ Y. Wang, B. J. Braams, J. M. Bowman, S. Carter, and D. P. Tew, J. Chem. Phys. 128, 224314 (2008).

${ }^{2}$ W. Rowe, R. Duerst, and E. Wilson, J. Am. Chem. Soc. 98, 4021 (1976).

${ }^{3}$ S. Baughcum, R. Duerst, W. Rowe, Z. Smith, and E. Wilson, J. Am. Chem. Soc. 103, 6296 (1981).

${ }^{4}$ S. Baughcum, Z. Smith, E. Wilson, and R. Duerst, J. Am. Chem. Soc. 106, 2260 (1984).

${ }^{5}$ P. Turner, S. L. Baughcum, S. L. Coy, and Z. Smith, J. Am. Chem. Soc. 106, 2265 (1984)

${ }^{6}$ D. W. Firth, K. Beyer, M. A. Dvorak, S. W. Reeve, A. Grushow, and K. R. Leopold, J. Chem. Phys. 94, 1812 (1991).

${ }^{7}$ T. Baba, T. Tanaka, I. Morino, K. M. T. Yamada, and K. Tanaka, J. Chem. Phys. 110, 4131 (1999).

${ }^{8}$ C. Seliskar and R. Hofmann, J. Mol. Spectrosc. 96, 146 (1982).
}

${ }^{9}$ Z. Smith, E. B. Wilson, and R. W. Duerst, Spectrochim. Acta, Part A 39, 1117 (1983).

${ }^{10}$ C. Duan and D. Luckhaus, Chem. Phys. Lett. 391, 129 (2004).

${ }^{11}$ T. N. Wassermann, D. Luckhaus, S. Coussan, and M. A. Suhm, Phys. Chem. Chem. Phys. 8, 2344 (2006).

${ }^{12}$ T. Carrington and W. H. Miller, J. Chem. Phys. 84, 4364 (1986).

${ }^{13}$ N. Shida, P. F. Barbara, and J. E. Almlöf, J. Chem. Phys. 91, 4061 (1989).

${ }^{14}$ N. Makri and W. H. Miller, J. Chem. Phys. 91, 4026 (1989).

${ }^{15}$ Z. Smedarchina, W. Siebrand, and M. Z. Zgierski, J. Chem. Phys. 103, 5326 (1995)

${ }^{16}$ T. D. Sewell, Y. Guo, and D. L. Thompson, J. Chem. Phys. 103, 8557 (1995).

${ }^{17}$ M. Ben-Nun and T. Martínez, J. Phys. Chem. A 103, 6055 (1999).

${ }^{18}$ V. A. Benderskii, E. V. Vetoshkin, I. S. Irgibaeva, and H. P. Trommsdorff, Chem. Phys. 262, 393 (2000).

${ }^{19}$ M. E. Tuckerman and D. Marx, Phys. Rev. Lett. 86, 4946 (2001).

${ }^{20}$ C. S. Tautermann, A. F. Voegele, T. Loerting, and K. R. Liedl, J. Chem. Phys. 117, 1962 (2002).

${ }^{21}$ C. S. Tautermann, A. F. Voegele, T. Loerting, and K. R. Liedl, J. Chem. Phys. 117, 1967 (2002).

${ }^{22}$ G. V. Mil'nikov, K. Yagi, T. Taketsugu, H. Nakamura, and K. Hirao, J. Chem. Phys. 119, 10 (2003).

${ }^{23}$ G. V. Mil'nikov, K. Yagi, T. Taketsugu, H. Nakamura, and K. Hirao, J. Comput. Phys. 120, 5036 (2004).

${ }^{24}$ K. Yagi, T. Taketsugu, and K. Hirao, J. Chem. Phys. 115, 10647 (2001).

${ }^{25}$ J. Ischtwan and M. A. Collins, J. Chem. Phys. 100, 8080 (1994).

${ }^{26}$ K. C. Thompson, M. J. T. Jordan, and M. A. Collins, J. Chem. Phys. 108, 564 (1998).

${ }^{27}$ M. D. Coutinho-Neto, A. Viel, and U. Manthe, J. Chem. Phys. 121, 9207 (2004).

${ }^{28}$ A. Viel, M. D. Coutinho-Neto, and U. Manthe, J. Chem. Phys. 126, 024308 (2007).

${ }^{29}$ D. Blume, M. Lewerenz, P. Niyaz, and K. B. Whaley, Phys. Rev. E 55, 3664 (1997)

${ }^{30}$ H.-D. Meyer, U. Manthe, and L. S. Cederbaum, Chem. Phys. Lett. 165, 73 (1990).

${ }^{31}$ U. Manthe, H.-D. Meyer, and L. S. Cederbaum, J. Chem. Phys. 97, 3199 (1992).

${ }^{32}$ B. Braams and J. M. Bowman, Int. Rev. Phys. Chem. 28, 577 (2009).

${ }^{33}$ D. P. Tew, N. C. Handy, and S. Carter, J. Chem. Phys. 125, 084313 (2006).

${ }^{34}$ U. Manthe, J. Chem. Phys. 128, 064108 (2008).

${ }^{35}$ M. H. Beck, A. Jäckle, G. A. Worth, and H.-D. Meyer, Phys. Rep. 324, 1 (2000).

${ }^{36}$ U. Manthe, J. Theor. Comput. Chem. 1, 153 (2002).

${ }^{37}$ H.-D. Meyer and G. A. Worth, Theor. Chem. Acc. 109, 251 (2003).

${ }^{38}$ F. Huarte-Larrañaga and U. Manthe, Z. Phys. Chem. 221, 171 (2007).

${ }^{39}$ D. O. Harris, G. G. Engerholm, and W. D. Gwinn, J. Chem. Phys. 43, 1515 (1965)

${ }^{40}$ A. S. Dickinson and P. R. Certain, J. Chem. Phys. 49, 4209 (1968).

${ }^{41}$ J. C. Light, I. P. Hamilton, and J. V. Lill, J. Chem. Phys. 82, 1400 (1985).

${ }^{42}$ D. Kosloff and R. Kosloff, J. Comput. Phys. 52, 35 (1983)

${ }^{43}$ U. Manthe, J. Chem. Phys. 105, 6989 (1996).

${ }^{44}$ U. Manthe and F. Matzkies, Chem. Phys. Lett. 252, 71 (1996).

${ }^{45}$ T. Gerdts and U. Manthe, J. Chem. Phys. 107, 6584 (1997).

${ }^{46}$ M. H. Beck and H.-D. Meyer, Z. Phys. D: At., Mol. Clusters 42, 113 (1997).

${ }^{47}$ U. Manthe, Chem. Phys. 329, 168 (2006).

${ }^{48}$ H. Wang and M. Thoss, J. Chem. Phys. 119, 1289 (2003).

${ }^{49}$ U. Manthe, J. Chem. Phys. 128, 164116 (2008).

${ }^{50}$ U. Manthe, J. Chem. Phys. 130, 054109 (2009).

${ }^{51}$ K. C. Thompson, M. J. T. Jordan, and M. A. Collins, J. Chem. Phys. 108, $8302(1998)$

${ }^{52}$ T. Wu, H.-J. Werner, and U. Manthe, Science 306, 2227 (2004).

${ }^{53}$ T. Wu, H.-J. Werner, and U. Manthe, J. Chem. Phys. 124, 164307 (2006). 